\title{
Comparative integromics on non-canonical WNT or planar cell polarity signaling molecules: Transcriptional mechanism of PTK7 in colorectal cancer and that of SEMA6A in undifferentiated ES cells
}

\author{
MASUKO KATOH ${ }^{1}$ and MASARU KATOH ${ }^{2}$ \\ ${ }^{1}$ M\&M Medical BioInformatics, Hongo 113-0033; ${ }^{2}$ Genetics and Cell Biology Section, \\ National Cancer Center Research Institute, Tokyo 104-0045, Japan
}

Received May 9, 2007; Accepted June 22, 2007

\begin{abstract}
Non-canonical WNT and planar cell polarity (PCP) are overlapping but distinct signaling pathways, which control convergent extension, neural tube closure, orientation of cilia and sensory hair cells, axon guidance, and cell motility. Non-canonical WNT signals, regulated by the interaction of WNT, WNT antagonist, Frizzled and ROR2, are transduced to JNK, ROCK, PKC, MAP3K7, and NFAT signaling cascades. PCP signals, regulated by the interaction of VANGL-PRICKLE complex, CELSR and Frizzled-DVL complex, are transduced to JNK, ROCK, and other uncharacterized signaling cascades. PTK7 signaling, regulated by SEMA6 and Plexin-A family members, affects PCP pathway through VANGL. Here, integrative genomic analyses on WNT5A, WNT5B, WNT11, FZD3, FZD6, ROR1, ROR2, RYK, CELSR1, CELSR2, CELSR3, VANGL1, VANGL2, PRICKLE1, PRICKLE2, PTK7, SEMA6A, SEMA6B, SEMA6C and SEMA6D were carried out. PTK7 and SEMA6A were expressed in undifferentiated embryonic stem (ES) cells, SEMA6A in endodermal progenitors, CELSR1, VANGL1 and PTK7 in gastrointestinal tumors. CELSR2, PRICKLE2 and SEMA6C were expressed in fetal brain, CELSR2, PRICKLE1 and SEMA6A in adult brain, WNT5A and CELSR3 in adult brain tumors. These facts indicate class switches of non-canonical WNT or PCP signaling molecules during embryogenesis and carcinogenesis. TCF/LEF-, SP1-, and 5 bHLH-binding sites within human $P T K 7$ promoter were conserved in chimpanzee, rhesus monkey, mouse, and rat PTK7 orthologs, which explained the mechanism of PTK7 upregulation in colorectal cancer. NANOG-, SOX2-, and POU5F1 (OCT3/OCT4)-binding sites
\end{abstract}

Correspondence to: Dr Masaru Katoh, Genetics and Cell Biology Section, National Cancer Center Research Institute, 5-1-1 Tsukiji, Chuo-ku, Tokyo 104-0045, Japan

E-mail: mkatoh-kkr@umin.ac.jp

Key words: WNT, frizzled, planar cell polarity, invasion, metastasis, bioinformatics, integrome network, systems medicine within intron 1 of the human SEMA6A gene were conserved in chimpanzee, rhesus monkey, mouse, and rat SEMA6A orthologs, which explained the mechanism of SEMA6A upregulation in undifferentiated ES cells. Most of noncanonical WNT or PCP signaling molecules, except PTK7 and $S E M A 6 A$, were not frequently expressed in undifferentiated human ES cells. Non-canonical WNT or PCP signaling pathway, activated to orchestrate gastrulation and neurulation, was relatively downregulated in undifferentiated ES cells derived from inner cell mass of blastocysts.

\section{Introduction}

WNT signals are transduced to the canonical pathway for the cell fate determination, and to the non-canonical pathway for the regulation of planar cell polarity (PCP), cell adhesion and motility in a context-dependent manner (1-3). Transcription of MYC, CCND1, FGF20, WISP1, JAG1, DKK1, and GCG genes are activated by the canonical WNT signals (4-11), while JNK, ROCK, PKC, MAP3K7 and NFAT signaling cascades are activated by the non-canonical WNT signals (12-14).

PCP signaling pathway is overlapping with the noncanonical WNT signaling pathway (Fig. 1A); however, PCP and non-canonical WNT are distinct signaling pathways. PCP signals, regulated by the interaction of VANGL-PRICKLE complex, CELSR and Frizzled-DVL complex, are transduced to JNK, ROCK, and other uncharacterized signaling cascades (14-17). PTK7 signaling, regulated by SEMA6 and Plexin-A family members, affects the PCP pathway through VANGL $(18,19)$. Non-canonical WNT or PCP signaling pathway controls convergent extension, neural tube closure, orientation of cilia and sensory hair cells, axon guidance, and cell motility (15-17,20-25).

We cloned and characterized WNT5B, WNT11, FZD3, FZD6, VANGL1, and VANGL2 using molecular biology techniques (26-32). We then identified and characterized PRICKLE1 and PRICKLE2 using bioinformatics/techint and human intelligence/humint (33). Other groups cloned and characterized WNT5A, ROR1, ROR2, RYK, CELSR1, CELSR2, CELSR3, PTK7, SEMA6A, SEMA6B, SEMA6C, and SEMA6D (34-41). 


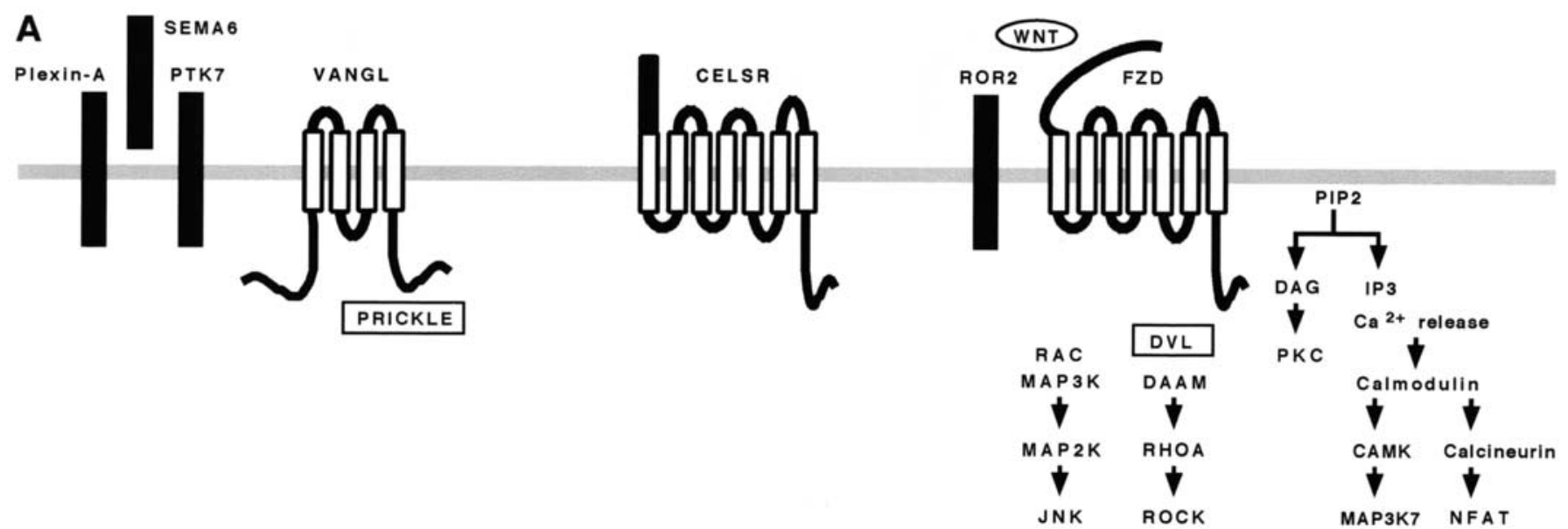

B

\begin{tabular}{|c|c|c|c|c|c|c|c|c|c|c|c|}
\hline & $\begin{array}{c}\text { Human } \\
\text { ES ce11s }\end{array}$ & $\underset{\substack{\text { Embryoid } \\
\text { body }}}{2}$ & $\begin{array}{c}\text { Neural } \\
\text { progenitors }\end{array}$ & $\begin{array}{l}\text { Fetal } \\
\text { brain }\end{array}$ & $\begin{array}{l}\text { Infant } \\
\text { brain }\end{array}$ & $\begin{array}{l}\text { Adult } \\
\text { brain }\end{array}$ & $\begin{array}{l}\text { Brain } \\
\text { tumors }\end{array}$ & $\begin{array}{l}\text { Endoderma } \\
\text { progenitors }\end{array}$ & $\begin{array}{l}\text { Gastric } \\
\text { s cancer }\end{array}$ & $\begin{array}{c}\text { Pancreatic } \\
\text { cancer }\end{array}$ & $\begin{array}{c}\text { Colorectal } \\
\text { cancer }\end{array}$ \\
\hline 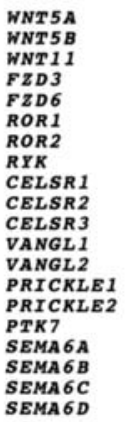 & $\begin{array}{r}1 \\
0 \\
0 \\
0 \\
4 \\
3 \\
2 \\
1 \\
6 \\
2 \\
0 \\
1 \\
6 \\
3 \\
1 \\
13 \\
27 \\
2 \\
1 \\
1\end{array}$ & $\begin{array}{r}7 \\
0 \\
0 \\
3 \\
3 \\
3 \\
1 \\
2 \\
1 \\
4 \\
1 \\
1 \\
7 \\
1 \\
4 \\
11 \\
1 \\
2 \\
1 \\
1\end{array}$ & $\begin{array}{l}2 \\
0 \\
0 \\
2 \\
0 \\
1 \\
0 \\
2 \\
3 \\
1 \\
0 \\
0 \\
4 \\
4 \\
1 \\
4 \\
5 \\
0 \\
2 \\
0\end{array}$ & $\begin{array}{r}0 \\
1 \\
7 \\
7 \\
2 \\
0 \\
0 \\
0 \\
5 \\
17 \\
6 \\
1 \\
9 \\
9 \\
27 \\
5 \\
9 \\
1 \\
13 \\
8\end{array}$ & $\begin{array}{r}1 \\
0 \\
0 \\
0 \\
0 \\
5 \\
0 \\
6 \\
1 \\
3 \\
1 \\
0 \\
2 \\
6 \\
13 \\
0 \\
2 \\
2 \\
1 \\
0\end{array}$ & $\begin{array}{r}2 \\
2 \\
1 \\
9 \\
11 \\
5 \\
1 \\
3 \\
0 \\
84 \\
7 \\
4 \\
3 \\
27 \\
9 \\
9 \\
31 \\
2 \\
6 \\
12\end{array}$ & $\begin{array}{r}10 \\
2 \\
0 \\
1 \\
0 \\
1 \\
1 \\
3 \\
0 \\
5 \\
10 \\
1 \\
6 \\
3 \\
4 \\
2 \\
1 \\
4 \\
3 \\
0\end{array}$ & $\begin{array}{r}0 \\
0 \\
0 \\
3 \\
2 \\
1 \\
3 \\
2 \\
2 \\
0 \\
1 \\
0 \\
0 \\
0 \\
0 \\
6 \\
13 \\
0 \\
1 \\
0\end{array}$ & $\begin{array}{l}1 \\
2 \\
1 \\
1 \\
2 \\
0 \\
2 \\
3 \\
1 \\
0 \\
2 \\
4 \\
0 \\
0 \\
2 \\
1 \\
1 \\
1 \\
0 \\
0\end{array}$ & $\begin{array}{l}0 \\
1 \\
0 \\
1 \\
0 \\
1 \\
1 \\
1 \\
6 \\
1 \\
1 \\
3 \\
1 \\
0 \\
1 \\
3 \\
0 \\
3 \\
1 \\
0\end{array}$ & $\begin{array}{l}2 \\
4 \\
1 \\
1 \\
1 \\
3 \\
1 \\
2 \\
2 \\
0 \\
1 \\
4 \\
1 \\
1 \\
0 \\
7 \\
0 \\
0 \\
3 \\
0\end{array}$ \\
\hline
\end{tabular}

Figure 1. Non-canonical WNT or PCP signaling pathway. (A) Schematic representation of non-canonical WNT or PCP signaling molecules. Non-canonical WNT signals, regulated by the interaction of WNT, Frizzled and ROR2, are transduced to JNK, ROCK, PKC, MAP3K7, and NFAT signaling cascades. PCP signals, regulated by the interaction of VANGL-PRICKLE complex, CELSR and Frizzled-DVL complex, are transduced to JNK, ROCK, and other uncharacterized signaling cascades. PTK7 signaling, regulated by SEMA6 and Plexin-A family members, affects PCP pathway through VANGL. Noncanonical WNT or PCP signaling pathway controls convergent extension, neural tube closure, orientation of cilia and sensory hair cells, axon guidance, and cell motility. (B) Expression profile. Number of ESTs derived from human genes encoding non-canonical WNT or PCP signaling molecules are listed up. PTK7 and SEMA6A are relatively frequently expressed in undifferentiated ES cells.

Here, expression analyses on WNT5A, WNT5B, WNT11, FZD3, FZD6, ROR1, ROR2, RYK, CELSR1, CELSR2, CELSR3, VANGL1, VANGL2, PRICKLE1, PRICKLE2, PTK7, SEMA6A, SEMA6B, SEMA6C, and SEMA6D were carried out. Most of these genes were preferentially expressed in fetal tissues, adult tissues, and tumors, while PTK7 and SEMA6A in undifferentiated human embryonic stem (ES) cells. Comparative genomic analyses on PTK7 and SEMA6A genes were further carried out to elucidate the mechanisms of PTK7 and SEMA6A expression.

\section{Materials and methods}

In silico expression analyses. Expressed sequence tags (ESTs) derived from human genes encoding non-canonical WNT or PCP signaling molecules were searched for using the BLAST programs as described previously (42-44). WNT5A RefSeq (NM_003392.3), WNT5B RefSeq (NM_030775.2), WNT11 RefSeq (NM_004626.2), FZD3 RefSeq (NM_017412.2), FZD6 RefSeq (NM_003506.2), ROR1 RefSeq (NM_005012.2), ROR2 RefSeq (NM 004560.2), RYK RefSeq (NM_001005861.2), CELSR1
RefSeq (NM_014246.1), CELSR2 RefSeq (NM_001408.1), CELSR3 RefSeq (NM_001407.2), VANGL1 cDNA (45), VANGL2 cDNA (45), PRICKLE1 RefSeq (NM_153026.1), PRICKLE2 RefSeq (NM_198859.2), PTK7 RefSeq (NM_002821.3), SEMA6A RefSeq (NM_020796.3), SEMA6B RefSeq (NM_032108.2), SEMA6C RefSeq (NM_030913.3), and SEMA6D RefSeq (NM_024966.2) were used as query sequences for the BLAST programs. The sources of human ESTs were listed up for in silico expression analyses.

Exon-intron structure of the human PTK7 and SEMA6A genes. Human genome sequences corresponding to PTK7 and SEMA6A genes were searched for with the BLAST programs as described previously (46-48). Exon-intron boundaries were determined based on the consensus sequence of exon-intron junctions ('gt ..... ag' rule of intronic sequence) and codon usage within the coding region.

Comparative genomics on the PTK7 and SEMA6A genes. Human genome sequences around the PTK7 and SEMA6A genes were compared with chimpanzee, rhesus monkey, 
A

BHLH BHLH BHLH BHLH SP1 TCF/EF BHLH

B

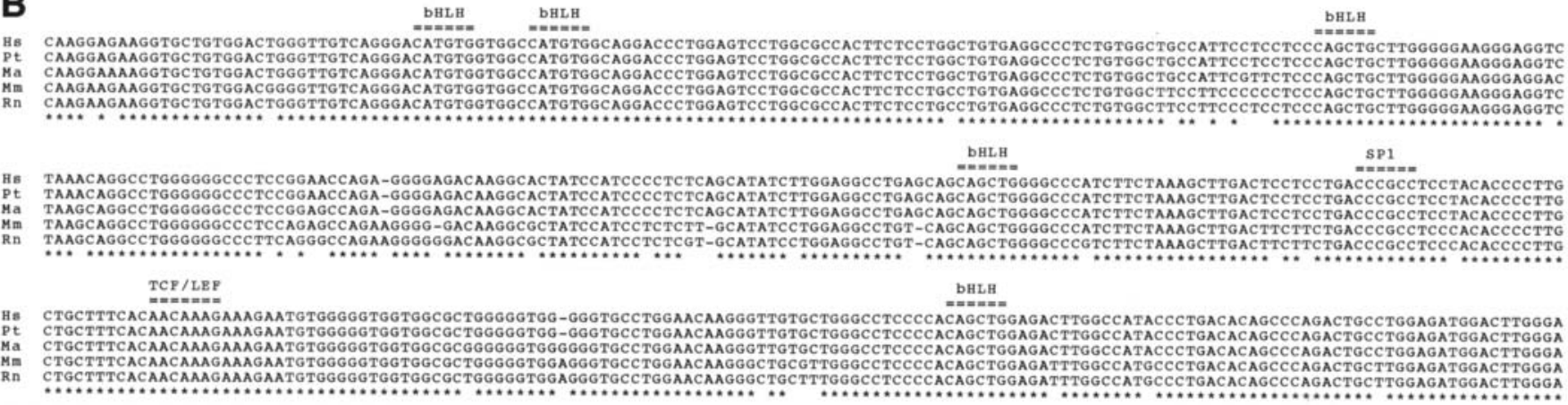

Figure 2. Comparative genomics on the PTK7 promoter. (A) Schematic representation of human PTK7 promoter region. Exon 1 is shown by an open box. Region highly conserved in mouse $P t k 7$ promoter is shown by a gray under bar. Conserved transcription factor-binding sites are also shown. (B) Alignment of conserved promoter region of the PTK7 orthologs. Hs, human; Pt, chimpanzee; Ma, rhesus monkey; Mm, mouse; Rn, rat. Conserved nucleotides are shown by asterisks below the alignment. Conserved bHLH-, SP1-, and TCF/LEF-binding sites are shown by a double overline above the alignment.

SEMAGA gene at human chromosome 5 q23.1

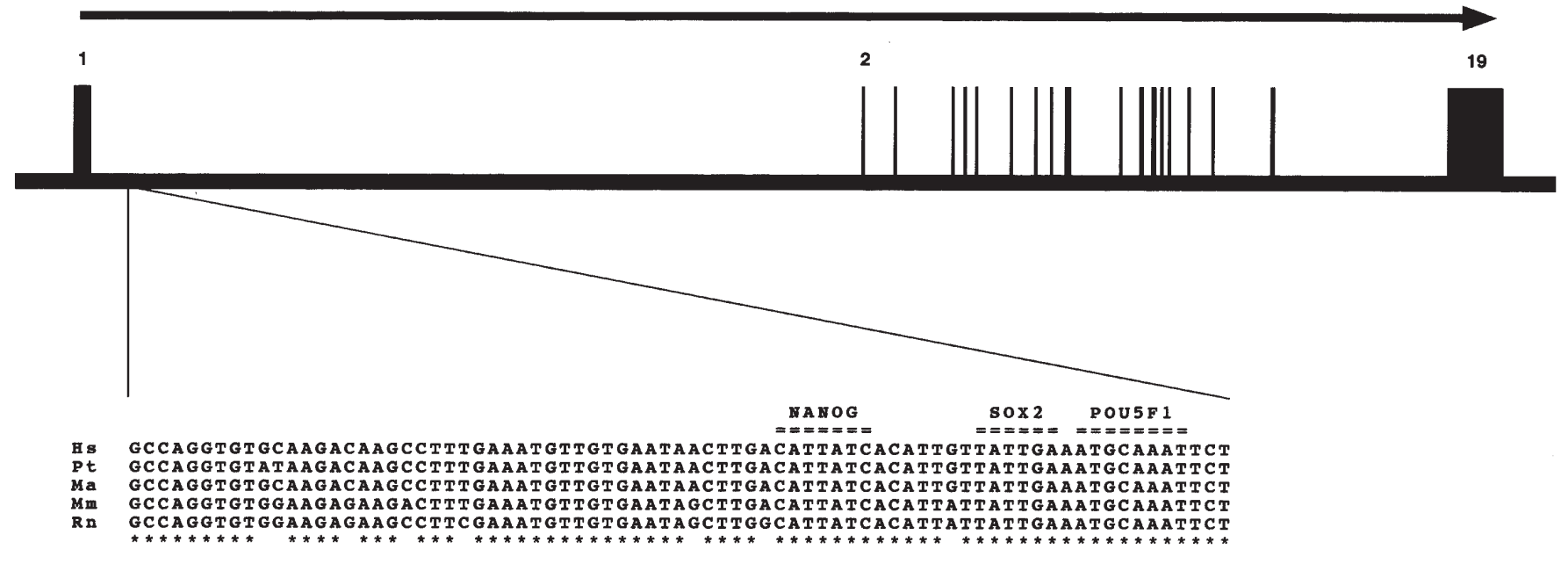

Figure 3. Comparative genomics on the SEMA6A gene. Schematic representation of human SEMA6A gene. Exons are shown by a closed box. Alignment of conserved region within intron 2 is also shown below the gene structure. Hs, human; Pt, chimpanzee; Ma, rhesus monkey; Mm, mouse; Rn, rat. Conserved nucleotides are shown by asterisks below the alignment. Conserved NANOG-, SOX2-, and POU5F1-binding sites are shown by a double overline above the alignment.

mouse and rat genome sequences using the BLAST programs. Transcription factor-binding sites within the evolutionarily conserved regions were then searched for using the Match program, Genetyx program, and the manual curation as described previously (49-51).

\section{Results}

Expression profile of non-canonical WNT or PCP signaling molecules. Sources of ESTs derived from human WNT5A, WNT5B, WNT11, FZD3, FZD6, ROR1, ROR2, RYK, CELSR1, CELSR2, CELSR3, VANGL1, VANGL2, PRICKLE1, PRICKLE2, PTK7, SEMA6A, SEMA6B, SEMA6C, and $S E M A 6 D$ mRNAs were listed up for the in silico expression analyses (Fig. 1B). PTK7 and SEMA6A were expressed in undifferentiated ES cells, SEMA6A in endodermal progenitors,
CELSR1, VANGL1 and PTK7 in gastrointestinal tumors. CELSR2, PRICKLE2 and SEMA6C were expressed in fetal brain, CELSR2, PRICKLE1 and SEMA6A in adult brain, $W N T 5 A$ and $C E L S R 3$ in adult brain tumors.

Because we have been working on the signaling network in ES cells and cancer stem/progenitor cells (50-53), comparative genomic analyses on PTK7 and SEMA6A genes were further carried out.

Comparative genomic analyses on PTK7 orthologs. BLAST programs revealed that the human PTK7 gene was located within AL355385.15 genome sequence, chimpanzee PTK7 gene within NW_001236528.1 genome sequence, rhesus monkey PTK7 gene within AC197857.4 genome sequence, mouse Ptk7 gene within AC165445.1 genome sequence, and rat Ptk7 gene within AC131887.3 genome sequence. 
Comparative genomic analyses revealed that the 5'-promoter region of the human $P T K 7$ gene, corresponding to nucleotide position -991 to -559 , were well conserved in chimpanzee, rhesus monkey, mouse, and rat PTK7 orthologs (Fig. 2A).

Transcription factor-binding sites within the conserved region of PTK7 5'-flanking promoter region were next searched for. Five bHLH-binding sites, SP1-binding site, and TCF/ LEF-binding site within human, chimpanzee, rhesus monkey, mouse, and rat PTK7 promoters were completely conserved (Fig. 2B).

Comparative genomic analyses on SEMA6A orthologs. BLAST programs revealed that the human SEMA6A gene was located within AC027304.4 and AC008524.6 genome sequences, chimpanzee SEMA6A gene within NW_001235419.1 genome sequence, rhesus monkey SEMA6A gene within AC169874.1 genome sequence, mouse Sema6a gene within AC121783.2 genome sequence, and rat Sema6a gene within AC094771.5 genome sequence.

Exon-intron structure of the human SEMA6A gene was determined based on the consensus sequence of exon-intron junctions. The human SEMA6A gene, consisting of 19 exons, was about $130 \mathrm{~kb}$ in size (Fig. 3). The first and last exons were larger than the other exons. Intron 1 was about $70 \mathrm{~kb}$ in size. Comparative genomic analyses revealed that the 5'promoter region and intron 1 of human SEMA6A gene was relatively well conserved in mouse Sema6a gene.

Transcription factor-binding sites within the conserved promoter regions and intron 1 of SEMA6A orthologs were next searched for. NANOG-, SOX2-, and POU5F1 (OCT3/ OCT4)-binding sites within intron 1 of human SEMA6A gene were completely conserved in chimpanzee, rhesus monkey, mouse, and rat SEMA6A orthologs (Fig. 3).

\section{Discussion}

Integrative genomic analyses on non-canonical WNT or PCP signaling molecules were carried out in this study. PTK7 and SEMA6A were expressed in undifferentiated ES cells, SEMA6A in endodermal progenitors, CELSR1, VANGL1 and PTK7 in gastrointestinal tumors (Fig. 1B). CELSR2, PRICKLE2 and SEMA6C were expressed in fetal brain, CELSR2, PRICKLE1 and SEMA6A in adult brain, WNT5A and CELSR3 in adult brain tumors (Fig. 1B). These facts indicate class switches of non-canonical WNT or PCP signaling molecules during embryogenesis and carcinogenesis.

Comparative genomic analyses on the PTK7 and SEMA6A genes were further carried out to elucidate the mechanisms of their expression in undifferentiated ES cells. Five bHLHbinding sites, SP1-binding site, and TCF/LEF-binding site within the human PTK7 5'-promoter region were conserved in chimpanzee, rhesus monkey, mouse, and rat PTK7 genes (Fig. 2). NANOG-, SOX2-, and POU5F1-binding sites within intron 1 of human SEMA6A gene were conserved in chimpanzee, rhesus monkey, mouse, and rat SEMA6A genes (Fig. 3).

PTK7 is a transmembrane protein with extracellular immunoglobulin-like domains and a cytoplasmic tyrosine kinase-like domain (39). PTK7 is homologous to ROR1, ROR2 and RYK receptor tyrosine kinases; however, PTK7 is a pseudokinase without detectable catalytic tyrosine kinase activity due to amino-acid substitution within the catalytic domain (54). PTK7, also known as CCK4, is upregulated in colorectal cancer. Because the canonical WNT signaling pathway is frequently activated in colorectal cancer, conserved TCF/LEF-binding site within the PTK7 promoter region explains the mechanism of $P T K 7$ upregulation in colorectal cancer.

SEMA6 family members are transmembrane-type ligands for Plexin-A family receptors to regulate axon guidance and cell motility $(18,19)$. SEMA6A is reported to bind to PlexinA2, Plexin-A4, while SEMA6C and SEMA6D to Plexin-A1. We also investigated the expression profile of Plexin-A family members to identify that Plexin-A1 was expressed in undifferentiated human ES cells (Katoh and Katoh, unpublished data). SEMA6D signal is transduced through the Plexin-A1 and PTK7 receptor complex for the activation of Rac signaling cascade rather than Rho signaling cascade (18). SEMA6A signal transduction through Plexin-A1 and PTK7 receptor complex in undifferentiated human ES cells should be investigated in the future.

Most of non-canonical WNT or PCP signaling molecules, except PTK7 and SEMA6A, were not frequently expressed in undifferentiated human ES cells. Non-canonical WNT or PCP signaling pathway, activated to orchestrate gastrulation and neurulation, was relatively downregulated in undifferentiated ES cells derived from the inner cell mass of blastocysts.

\section{References}

1. Katoh M and Katoh M: WNT signaling pathway and stem cell signaling network. Clin Cancer Res (In press).

2. Swain RK, Katoh M, Medina A and Steinbeisser H: Xenopus frizzled-4S, a splicing variant of Xfz4, is a context-dependent activator and inhibitor of Wnt/ß-catenin signaling. Cell Commun Signal 3: 12, 2005.

3. Katoh M and Katoh M: STAT3-induced WNT5A signaling loop in embryonic stem cells, adult normal tissues, chronic persistent inflammation, rheumatoid arthritis, and cancer. Int J Mol Med 19: 273-278, 2007.

4. He TC, Sparks AB, Rago C, et al: Identification of $c-M Y C$ as a target of the APC pathway. Science 281: 1509-1512, 1998.

5. Tetsu $\mathrm{O}$ and McCormick F: $\beta$-catenin regulates expression of cyclin D1 in colon carcinoma cells. Nature 398: 422-426, 1999.

6. Katoh $\mathrm{M}$ and Katoh M: Comparative genomics on FGF20 orthologs. Oncol Rep 14: 287-290, 2005.

7. Katoh M and Katoh M: Crosstalk of WNT and FGF signaling pathways at GSK3ß to regulate B-catenin and SNAIL signaling cascades. Cancer Biol Ther 5: 1059-1064, 2006.

8. Pennica D, Swanson TA, Welsh JW, et al: WISP genes are members of the connective tissue growth factor family that are up-regulated in Wnt1-transformed cells and aberrantly expressed in human colon tumors. Proc Natl Acad Sci USA 95: 14717-14722, 1998.

9. Katoh M and Katoh M: Notch ligand, JAG1, is evolutionarily conserved target of canonical WNT signaling pathway in progenitor cells. Int J Mol Med 17: 681-685, 2006.

10. Chamorro MN, Schwartz DR, Vonica A, et al: FGF20 and $D K K 1$ are transcriptional target of $\beta$-catenin and FGF20 is implicated in cancer and development. EMBO J 24: 73-84, 2005.

11. Yi F, Brubaker PL and Jin T: TCF-4 mediates cell type-specific regulation of proglucagon gene expression by $\beta$-catenin and glycogen synthase kinase-3ß. J Biol Chem 280: 1457-1464, 2005.

12. Boutros M, Paricio N, Strutt DI, et al: Dishevelled activates JNK and discriminates between JNK pathways in planar polarity and wingless signaling. Cell 94: 109-118, 1998. 
13. Dejmek J, Safholm A, Kamp Nielsen C, et al: Wnt-5a/Ca ${ }^{2+}$ induced NFAT activity is counteracted by Wnt-5a/Yes-Cdc42casein kinase Ia signaling in human mammary epithelial cells. Mol Cell Biol 26: 6024-6036, 2006.

14. Katoh M: WNT/PCP signaling pathway and human cancer. Oncol Rep 14: 1583-1588, 2005.

15. Myers DC, Sepich DS and Solnica-Krezel L: Convergence and extension in vertebrate gastrulae: cell movements according to or in search of identity. Trends Genet 18: 447-455, 2002.

16. Keller R: Shaping the vertebrate body plan by polarized embryonic cell movements. Science 298: 1950-1954, 2002

17. Montcouquiol M, Rachel RA, Lanford PJ, et al: Identification of Vangl2 and Scribl as planar polarity genes in mammals. Nature 423: 173-177, 2003.

18. Toyofuku T, Zhang H, Kumanogoh A, et al: Dual roles of Sema6D in cardiac morphogenesis through region-specific association of its receptor, Plexin-A1, with off-track and vascular endothelial growth factor receptor type 2. Genes Dev 18: 435-447, 2004.

19. Suto F, Ito K, Uemura M, et al: Plexin-a4 mediates axonrepulsive activities of both secreted and transmembrane semaphorins and plays roles in nerve fiber guidance. J Neurosci 25: 3628-3637, 2005 .

20. Guo N, Hawkins C and Nathans J: Frizzled6 controls hair patterning in mice. Proc Natl Acad Sci USA 101: 9277-9281, 2004.

21. Lu W, Yamamoto V, Ortega B and Baltimore D: Mammalian Ryk is a Wnt coreceptor required for stimulation of neurite outgrowth. Cell 119: 97-108, 2004.

22. Curtin JA, Quint E, Tsipouri V, et al: Mutation of Celsr1 disrupts planar polarity of inner ear hair cells and causes severe neural tube defects in the mouse. Curr Biol 13: 1129-1133, 2003.

23. Lu X, Borchers AG, Jolicoeur C, et al: PTK7/CCK-4 is a novel regulator of planar cell polarity in vertebrates. Nature 430: 93-98, 2004.

24. Saitoh T and Katoh M: Frequent up-regulation of WNT5A mRNA in primary gastric cancer. Int J Mol Med 9: 515-519, 2002.

25. Weeraratna AT, Jiang Y, Hostetter G, et al: Wnt5a signaling directly affects cell motility and invasion of metastatic melanoma. Cancer Cell 1: 279-288, 2002.

26. Saitoh T and Katoh M: Molecular cloning and characterization of human $W N T 5 B$ on chromosome 12 p 13.3 region. Int J Oncol 19: 347-351, 2001 .

27. Kirikoshi H, Sekihara H and Katoh M: Molecular cloning and characterization of human WNT11. Int J Mol Med 8: 651-656, 2001 .

28. Kirikoshi H, Koike J, Sagara N, Saitoh T, Tokuhara M, Tanaka K, Sekihara H, Hirai M and Katoh M: Molecular cloning and genomic structure of human Frizzled-3 at chromosome 8p21. Biochem Biophys Res Commun 271: 8-14, 2000.

29. Tokuhara M, Hirai M, Atomi Y, Terada M and Katoh M: Molecular cloning and characterization of human Frizzled-6. Biochem Biophys Res Commun 243: 622-627, 1998.

30. Katoh M: Molecular cloning and characterization of Strabismus 2 (STB2). Int J Oncol 20: 993-998, 2002

31. Katoh M: Structure and expression of Strabismus 1 (STB1) gene on human chromosome 1q21-q23. Int J Oncol 20: 1197-1203, 2002.

32. Katoh M: Strabismus (STB)/Vang-like (Vangl) gene family. Int J Mol Med 10: 11-15, 2002

33. Katoh $\mathrm{M}$ and Katoh $\mathrm{M}$ : Identification and characterization of human PRICKLE1 and PRICKLE2 genes as well as mouse Prickle1 and Prickle 2 genes homologous to Drosophila tissue polarity gene prickle. Int J Mol Med 11: 249-256, 2003.

34. Clark CC, Cohen I, Eichstetter I, et al: Molecular cloning of the human proto-oncogene Wnt-5A and mapping of the gene (WNT5A) to chromosome 3p14-p21. Genomics 18: 249-260, 1993.
35. Masiakowski P and Carroll RD: A novel family of cell surface receptors with tyrosine kinase-like domain. J Biol Chem 267: 26181-26190, 1992.

36. Stacker SA, Hovens CM, Vitali A, et al: Molecular cloning and chromosomal localisation of the human homologue of a receptor related to tyrosine kinases (RYK). Oncogene 8: 1347-1356, 1993.

37. Hadjantonakis AK, Sheward WJ, Harmar AJ, et al: Celsr1, a neural-specific gene encoding an unusual seven-pass transmembrane receptor, maps to mouse chromosome 15 and human chromosome 22qter. Genomics 45: 97-104, 1997.

38. $\mathrm{Wu} \mathrm{Q}$ and Maniatis T: A striking organization of a large family of human neural cadherin-like cell adhesion genes. Cell 97 : 779-790, 1999

39. Mossie K, Jallal B, Alves F, et al: Colon carcinoma kinase-4 defines a new subclass of the receptor tyrosine kinase family. Oncogene 11: 2179-2184, 1995.

40. Correa RG, Sasahara RM, Bengtson MH, et al: Human semaphorin $6 \mathrm{~B}$, a novel human class 6 semaphorin gene: alternative splicing and all-trans-retinoic acid-dependent downregulation in glioblastoma cell lines. Genomics 73: 343-348, 2001.

41. Qu X, Wei H, Zhai Y, et al: Identification, characterization, and functional study of the two novel human members of the semaphorin gene family. J Biol Chem 277: 35574-35585, 2002.

42. Katoh M: Paradignshift in gene-finding method: From benchtop approach to desk-top approach. Int J Mol Med 10: 677-682, 2002.

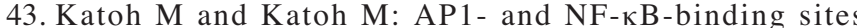
conserved among mammalian $W N T 10 B$ orthologs elucidate the TNF $\alpha$-WNT10B signaling loop implicated in carcinogenesis and adipogenesis. Int J Mol Med 19: 699-703, 2007.

44. Katoh $\mathrm{M}$ and Katoh M: Comparative integromics on FZD7 orthologs: Conserved binding sites for PU.1, SP1, CCAAT-box and $\mathrm{TCF} / \mathrm{LEF} / \mathrm{SOX}$ transcription factors within 5'promoter region of mammalian FZD7 orthologs. Int J Mol Med 19: 529-533, 2007.

45. Katoh Y and Katoh M: Comparative genomics on VANGL1 and VANGL2 genes. Int J Oncol 26: 1435-1440, 2005.

46. Katoh $M$ and Katoh $M$ : Identification and characterization of human FMNL1, FMNL2 and FMNL3 genes in silico. Int J Oncol 22: $1161-1168,2003$

47. Katoh $M$ and Katoh M: Identification and characterization of human BCL9L gene and mouse Bcl9l gene in silico. Int J Mol Med 12: 643-649, 2003

48. Katoh $\mathrm{M}$ and Katoh M: Identification and characterization of human HES2, HES3, and HES5 genes in silico. Int J Oncol 25: 529-534, 2004

49. Katoh $\mathbf{M}$ and Katoh M: WNT antagonist, DKK2, is Notch signaling target in intestinal stem cells: Augmentation of negative regulation system for canonical WNT signaling pathway by Notch-DKK2 signaling loop in primates. Int J Mol Med 19: 197-201, 2007

50. Katoh Y and Katoh M: Conserved POU-binding site linked to SP1-binding site within FZD5 promoter: Transcriptional mechanisms of FZD5 in undifferentiated human ES cells, fetal liver/spleen, adult colon, pancreatic islet, and diffuse-type gastric cancer. Int J Oncol 30: 751-755, 2007.

51. Katoh $\mathrm{M}$ and Katoh $\mathrm{M}$ : Conserved POU/OCT- and GATAbinding sites in 5'-flanking promoter region of mammalian $W N T 8 B$ orthologs. Int J Oncol 30: 1273-1277, 2007.

52. Katoh M: Networking of WNT, FGF, Notch, BMP, and Hedgehog signaling pathways during carcinogenesis. Stem Cell Rev (In press)

53. Katoh M: Dysregulation of stem cell signaling network due to germline mutation, SNP, Helicobacter pylori infection, epigenetic change, and genetic alteration in gastric cancer. Cancer Biol Ther (In press).

54. Boudeau J, Miranda-Saavedra D, Barton GJ and Alessi DR: Emerging roles of pseudokinases. Trends Cell Biol 16: 443-452, 2006. 